\title{
AN INDEX TO SELECTED SCIENTIFIC NAMES IN EDWARDS' (1929) MONOGRAPH ON BRITISH CHIRONOMIDAE
}

\author{
By Martin Spies \\ Munich, Germany (e-mail: spies@zi.biologie.uni-muenchen.de)
}

\begin{abstract}
Introduction
Much critical information from historic publications and collections still awaits adequate incorporation into the chironomid system (see, e.g., SPIES 2001, SPIES \& SÆTHER 2004). The classic works by EDWARDS (1929) and GOETGHEBUER \& LENZ in LINDNER ("Die Fliegen der Palaearktischen Region") have been most influential, both as overviews of the earlier literature and by their original contributions. Unfortunately, access to and evaluation of their individual observations and proposals have been impeded by the index in EDWARDS (1929) being limited to some of the occurrences of genus-group names only, and by the absence of any indexes from the LINDNER volumes on Orthocladiinae (Goetghebuer 1940-1950) and Chironominae (GOETGHEBUER \& LENZ 1937-1962).
\end{abstract}

To remove the first of these obstacles, an index to scientific species-group names in Edwards (1929) is presented below. The missing indexes to LINDNER volumes are in preparation; they will be announced and made available upon completion.

\section{Methods}

The index entries below are mere references to data in EDWARDS (1929); they must not be used for other purposes without checking them against an appropriate source. Unless specifically stated, no entry implies any opinion of mine on nomenclatural status or taxonomic validity.

Modifications to the indexed data are made for conciseness only. In EDWARDS (1929), many names occur in more than a single respective spelling or genus-species combination. The index reduces such variation where possible without loss of critical information. For example, the entry $>$ falcigera / Kieffer / Pelopia / ... $/<$ also covers "P. falciger" (EDWARDS 1929: 288), whereas the variant original spellings Chironomus nigronitans and $C$. nigronitens are indexed separately.

Names EDWARDS (1929) used for 'varieties' are indexed without distinction from species names because under the rules of nomenclature they now apply to subspecies or species.

In the "Authorship" column, the term $>$ [author] misid. [author] $<$ means that EDWARDS deemed the name misapplied by the subsequent author(s); $>$ sensu [author] < denotes a subsequent use not conclusively evaluated against the original one; $>$ nec $<$ signals a junior homonym.

The far right column lists pages in EDWARDS (1929) containing relevant information on the corresponding taxon or name. However, not all occurrences of all names are indexed; e.g. those in comparative descriptions ('wing as in [species]') are omitted unless of special significance.

Page numbers in regular type may represent more than one mention of the name. Numbers in boldface refer to taxonomic descriptions. Suffixes $>\mathrm{f}<$ and $>\mathrm{k}<$ indicate that the name occurs in a figure caption or key, respectively.

Index to species-group names of Chironomidae in Edwards (1929)

\begin{tabular}{|l|l} 
Species-group name & Authorship \\
abbreviatus & Kieffer \\
abdominalis & Staeger \\
abstrusus & Kieffer \\
acuticornis & Kieffer \\
acutus & Kieffer \\
adjunctus & Walker \\
aestivalis & Goetghebuer \\
aestivus & Curtis \\
affinis & Walker \\
agrayloides & Kieffer \\
albicornis & Goetghebuer \\
albicornis & Meigen \\
albiforceps & Goetghebuer \\
albiforceps & Kieffer
\end{tabular}

\begin{tabular}{|l|} 
Genus in original \\
combination \\
Tendipes \\
Chironomus \\
Glyptotendipes \\
Corynoneura \\
Polypedilum \\
Chironomus \\
Metriocnemus \\
Chironomus \\
Chironomus \\
Tanytarsus \\
Cricotopus \\
Chironomus \\
Tanytarsus \\
Chironomus
\end{tabular}

$\left|\begin{array}{l}\text { Genus-group name(s) } \\ \text { stated or implied by Edwards } \\ \text { Chironomus (Microtendipes) } \\ \text { Pentapedilum (Phaenopsectra) } \\ \text { Chironomus (Glyptotendipes) } \\ \text { Corynoneura (Corynoneura) } \\ \text { Chironomus (Polypedilum) } \\ \text { Metriocnemus } \\ \text { Metriocnemus } \\ \text { Eurycnemus } \\ \text { Tanytarsus (Tanytarsus) } \\ \text { Chironomus (Lauterborniella) } \\ \text { Cricotopus } \\ \text { Chironomus (Polypedilum) } \\ \text { Pentapedilum (Phaenopsectra) } \\ \text { Chironomus (Chironomus) }\end{array}\right|$

Page numbers

397

375

392

369

398f, 402k, 404

311

313

309

410

405

351

402k, 403

375

387 


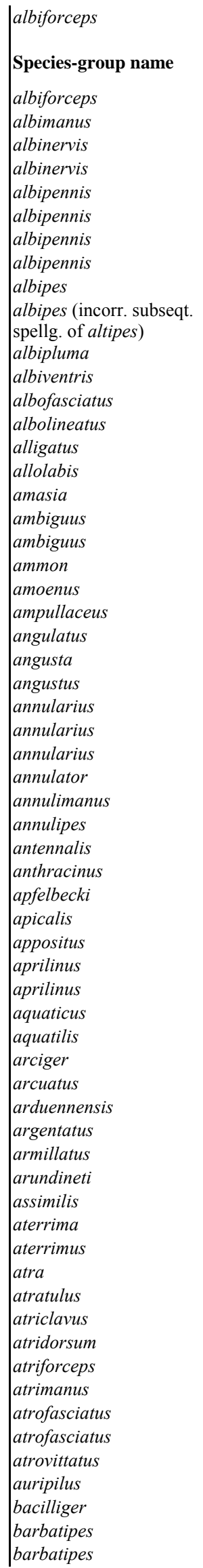

\begin{tabular}{|c|c|c|}
\hline Authorship & $\begin{array}{l}\text { Trichocladius } \\
\text { Genus in original } \\
\text { combination }\end{array}$ & $\begin{array}{l}\text { Cricotopus } \\
\text { Genus-group name(s) } \\
\text { stated or implied by Edwards }\end{array}$ \\
\hline Kieffer misid. Goet. & Trichocladius & Cricotopus \\
\hline Meigen & Chironomus & Chironomus (Paratendipes) \\
\hline Kieffer & Procladius & Procladius (Psilotanypus) \\
\hline van der Wulp & Orthocladius & Spaniotoma (Psectrocladius) \\
\hline Goetghebuer & Dactylocladius & Spaniotoma (Smittia) \\
\hline Goetghebuer & Dactylocladius & Spaniotoma (Smittia) \\
\hline Kieffer & Syndiamesa & Syndiamesa \\
\hline Meigen & Chironomus & Chironomus (Endochironomus) \\
\hline Kieffer & Iscocladius & Isocladius \\
\hline Zetterstedt & Chironomus & Pentapedilum (Phaenopsectra) \\
\hline Kieffer & Cricotopus & Cricotopus \\
\hline Kieffer & Lenzia & Pentapedilum (Phaenopsectra) \\
\hline Staeger & Chironomus & Chironomus (Chironomus) \\
\hline Meigen & Chironomus & Metriocnemus \\
\hline Walker & Chironomus & Metriocnemus \\
\hline Kieffer & Chironomus & Chironomus (Chironomus) \\
\hline Meigen & Chironomus & Cricotopus \\
\hline Goetghebuer & Graceus & Graceus \\
\hline van der Wulp & Chironomus & Chironomus \\
\hline Haliday & Diamesa & Diamesa \\
\hline Meigen & Chironomus & Cricotopus \\
\hline Kieffer & Metriocnemus & Metriocnemus \\
\hline Goetghebuer & Metriocnemus & Metriocnemus \\
\hline Edwards & Spaniotoma & Spaniotoma (Smittia) \\
\hline Goetghebuer & Cricotopus & Cricotopus \\
\hline De Geer & Tipula & Chironomus (Chironomus) \\
\hline De Geer misid. Goet. & Chironomus & Chironomus (Chironomus) \\
\hline De Geer misid. Verr./Coll. & Chironomus & Chironomus (Glyptotendipes) \\
\hline Goetghebuer & Cricotopus & Cricotopus \\
\hline Goetghebuer & Chironomus & Chironomus (Glyptotendipes) \\
\hline Meigen & Chironomus & Cricotopus \\
\hline Kieffer & Corynoneura & Bauseia \\
\hline Zetterstedt & Chironomus & Chironomus (Chironomus) \\
\hline Strobl & Chironomus & Chironomus (Polypedilum) \\
\hline Kieffer & Metriocnemus & Spaniotoma (Orthocladius) \\
\hline Walker & Chironomus & Tanytarsus (Micropsectra) \\
\hline Meigen misid. Kieffer & Chironomus & Chironomus (Chironomus) \\
\hline Meigen sensu Goet. & Chironomus & Chironomus (Chironomus) \\
\hline Kieffer sensu Goet. & Camptocladius & Spaniotoma (Smittia) \\
\hline Goetghebuer & Camptocladius & Spaniotoma (Smittia) \\
\hline Kieffer & Metriocnemus & Metriocnemus \\
\hline Goetghebuer & Chironomus & Chironomus (Chironomus) \\
\hline Goetghebuer & Tanytarsus & Tanytarsus (Tanytarsus) \\
\hline Goetghebuer & Dactylocladius & Spaniotoma (Orthocladius) \\
\hline Staeger & Chironomus & Chironomus (Chironomus) \\
\hline Goetghebuer & Tendipes & Chironomus (Polypedilum) \\
\hline Zetterstedt & Chironomus & Chironomus (Stictochironomus) \\
\hline Meigen & Chironomus & Spaniotoma (Smittia) \\
\hline Meigen & Chironomus & Camptocladius \\
\hline Winnertz & Corynoneura & Corynoneura (Corynoneura) \\
\hline Zetterstedt & Chironomus & Metriocnemus \\
\hline Kieffer & Metriocnemus & Metriocnemus \\
\hline Kieffer & Cladotanytarsus & Tanytarsus (Tanytarsus) \\
\hline Goetghebuer & Chironomus & Chironomus (Chironomus) \\
\hline Kieffer & Trichocladius & Cricotopus \\
\hline Kieffer & Camptochironomus & Chironomus (Chironomus) \\
\hline Kieffer & Tanytarsus & Tanytarsus (Micropsectra) \\
\hline Kieffer & Limnochironomus & Chironomus (Chironomus) \\
\hline Goetghebuer & Metriocnemus & Metriocnemus \\
\hline Kieffer & Cryptochironomus & Chironomus (Chironomus) \\
\hline Kieffer & Pelopia & Pentaneura \\
\hline Kieffer misid. Goet. & Pelopia & Pentaneura \\
\hline
\end{tabular}

$320 \mathrm{k}, 321$

Page numbers

321

395

302

334

$363 \mathrm{k}, 365$

363k, 365

303

394, 380

318

375

351

375

388

310

311

387

319

281, 374f, 377

378

307

319

314

314

$352 \mathrm{f}, 363 \mathrm{k}, 364,368 \mathrm{f}, 428 \mathrm{f}$

322,323

384,392

$383 \mathrm{k}, 384$

392

323

393

323

367

379f, 383, 383k

398f, 401k, 402, 429f

308, 339f, 347, 347k, 428f

407

382

$383 \mathrm{k}, 384$

360

361

315

389

412k, 414

337

388

379f, 402k, 403

401

360, 360k

357

369

$311 \mathrm{k}, 312$

$311 \mathrm{k}, 311$

417f, 418

390

318,321

382

408

386

311

389

291

291 


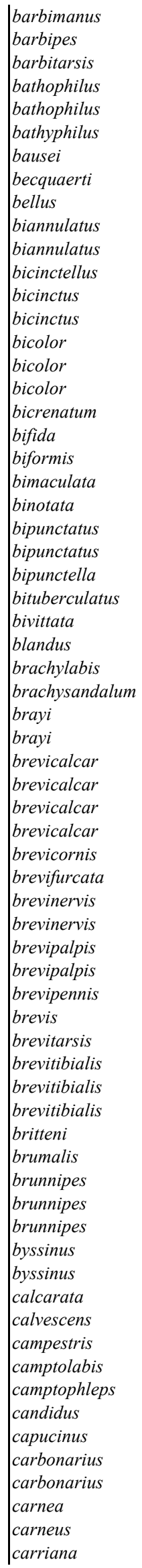

\begin{tabular}{|c|c|}
\hline Edwards & Spaniotoma \\
\hline Staeger & Chironomus \\
\hline Zetterstedt & Tanypus \\
\hline Kieffer & Tanytarsus \\
\hline Kieffer & Tendipes \\
\hline Kieffer & Procladius \\
\hline Kieffer & Tanytarsus \\
\hline Goetghebuer & Chironomus \\
\hline Loew & Tanypus \\
\hline Staeger & Chironomus \\
\hline Staeger misid. Goet. & Chironomus \\
\hline Goetghebuer & Cricotopus \\
\hline Goetghebuer & Tanytarsus \\
\hline Meigen & Chironomus \\
\hline Kieffer & Clunio \\
\hline Meigen & Chironomus \\
\hline Zetterstedt & Chironomus \\
\hline Kieffer & Polypedilum \\
\hline Kieffer & Metriocnemus \\
\hline Edwards & Cricotopus \\
\hline Kieffer & Isoplastus \\
\hline Wiedemann & Chironomus \\
\hline Kieffer & Chironomus \\
\hline Kieffer & Tanytarsus \\
\hline Zetterstedt & Chironomus \\
\hline Edwards & Tanytarsus \\
\hline Philippi & Spaniotoma \\
\hline van der Wulp & Chironomus \\
\hline Edwards & Chironomus \\
\hline Kieffer & Microtendipes \\
\hline Goetghebuer & Chironomus \\
\hline Goetghebuer & Tanytarsus \\
\hline Kieffer & Dactylocladius \\
\hline Kieffer & Dactylocladius \\
\hline Kieffer & Psectrotanypus \\
\hline Kieffer & Psectrotanypus \\
\hline Kieffer & Orthocladius \\
\hline Edwards & Orthocladius \\
\hline Kieffer & Corynoneura \\
\hline Malloch & Dactylocladius \\
\hline Kieffer & Cricotopus \\
\hline Kieffer & Trissocladius \\
\hline Holmgren & Chironomus \\
\hline Edwards & Tanytarsus \\
\hline Edwards & Metriocnemus \\
\hline Goetghebuer & Tanypus \\
\hline Zetterstedt & Chironomus \\
\hline Zetterstedt misid. Goet. & Chironomus \\
\hline Edwards & Chironomus \\
\hline Edwards & Metriocnemus \\
\hline Goetghebuer & Trichocladius \\
\hline Zetterstedt & Chironomus \\
\hline Zetterstedt sensu Edw. & Chironomus \\
\hline Schrank & Tipula \\
\hline Schrank & Tipula \\
\hline Edwards & Spaniotoma \\
\hline Edwards & Spaniotoma \\
\hline Edwards & Diamesa \\
\hline Kieffer & Tendipes \\
\hline Edwards & Spaniotoma \\
\hline Kieffer & Chironomus \\
\hline Zetterstedt & Chironomus \\
\hline Meigen & Chironomus \\
\hline Meigen misid. Goet. & Chironomus \\
\hline Fabricius & Chironomus \\
\hline Fabricius misid. Goet. & Chironomus \\
\hline Edwards & Corynoneura \\
\hline
\end{tabular}

\begin{tabular}{|c|c|}
\hline Spaniotoma (Psectrocladius) & $332 k, 333$ \\
\hline Chironomus (Glyptotendipes) & 391k, 392 \\
\hline Pentaneura & 292,293 \\
\hline Tanytarsus (Tanytarsus) & 411 \\
\hline Chironomus (Chironomus) & 383 \\
\hline Procladius (Psilotanypus) & 302 \\
\hline Tanytarsus (Stempellina) & 419,419 \\
\hline Chironomus (Chironomus) & 385 \\
\hline Psilotanypus & 300 \\
\hline Chironomus (Chironomus) & 388 \\
\hline Chironomus (Chironomus) & 388 \\
\hline Cricotopus & 321 \\
\hline Tanytarsus (Tanytarsus) & 415 \\
\hline Cricotopus & $318,320 \mathrm{k}, 321,322 \mathrm{f}$ \\
\hline Clunio & 371 \\
\hline Chironomus & 351 \\
\hline Spaniotoma (Eukiefferiella) & $351,351,351 \mathrm{k}$ \\
\hline Polypedilum & 403 \\
\hline Brillia & 310 \\
\hline Cricotopus & $322 \mathrm{f}, 324 \mathrm{k}, 325$ \\
\hline Anatopynia & 298 \\
\hline Pentaneura & $288,293,294$ \\
\hline Chironomus (Chironomus) & 390 \\
\hline Tanytarsus (Tanytarsus) & 415 \\
\hline Spaniotoma (Orthocladius) & $340 \mathrm{k}, 341$ \\
\hline Tanytarsus (Lundstroemia) & 408, $412 \mathrm{k}$ \\
\hline Spaniotoma & 327 \\
\hline Chironomus (Polypedilum) & 401,404 \\
\hline Chironomus (Lauterborniella) & 398f, 406 \\
\hline Chironomus (Microtendipes) & 397 \\
\hline Chironomus (Kribioxenus) & 396 \\
\hline Tanytarsus (Tanytarsus) & 414 \\
\hline Dactylocladius & 350 \\
\hline Spaniotoma (Eukiefferiella) & $351 \mathrm{k}, 352 \mathrm{f}, 353$ \\
\hline Psectrotanypus & 297 \\
\hline Anatopynia & 298 \\
\hline Orthocladius & 335,336 \\
\hline Spaniotoma (Smittia) & $363 \mathrm{k}, \mathbf{3 6 5}, 428 \mathrm{f}$ \\
\hline Corynoneura (Corynoneura) & 368 \\
\hline Dactylocladius & 351 \\
\hline Cricotopus & 318 \\
\hline Trissocladius & 309 \\
\hline Camptocladius & 357 \\
\hline Tanytarsus (Stempellina) & $417 f, 420,429 f$ \\
\hline Metriocnemus & 316 \\
\hline Pentaneura & 293, 294 \\
\hline Chironomus (Chironomus) & 386 \\
\hline Chironomus (Chironomus) & 386 \\
\hline Chironomus (Microtendipes) & 397k, 398f, 399 \\
\hline Metriocnemus & $314 f, 316$ \\
\hline Trichocladius & 326 \\
\hline Tanytarsus (Micropsectra) & 407 \\
\hline Tanytarsus (Micropsectra) & 374f, 407, 408 \\
\hline Spaniotoma (Smittia) & 335,362 \\
\hline Camptocladius & 355,357 \\
\hline Spaniotoma (Psectrocladius) & $332 \mathrm{k}, 333$ \\
\hline Spaniotoma (Eukiefferiella) & $351 \mathrm{k}, 352 \mathrm{f}, 353,368 \mathrm{f}$ \\
\hline Diamesa & $306 f, 307$ \\
\hline Chironomus (Chironomus) & $381 f, 387$ \\
\hline Spaniotoma (Eukiefferiella) & $351 \mathrm{k}, 352 \mathrm{f}, 353,368 \mathrm{f}, 428 \mathrm{f}$ \\
\hline Chironomus (Glyptotendipes) & 393 \\
\hline Cardiocladius & $282,317,346,370 f, 428 f$ \\
\hline Chironomus & 332 \\
\hline Spaniotoma (Psectrocladius) & 332 \\
\hline Pentaneura & 289,291 \\
\hline Pentaneura & 291 \\
\hline Corynoneura (Corynoneura) & 368f, 369, 429f \\
\hline
\end{tabular}




\begin{tabular}{|c|c|c|c|}
\hline Species-group name & $\begin{array}{l}\text { Kieffer } \\
\text { Authorship }\end{array}$ & $\begin{array}{l}\text { Metriocnemus } \\
\text { Genus in original } \\
\text { combination }\end{array}$ & $\begin{array}{l}\text { Metriocnemus } \\
\text { Genus-group name(s) } \\
\text { stated or implied by Edwards }\end{array}$ \\
\hline celeripes & Winnertz & Corynoneura & Corynoneura (Corynoneura) \\
\hline celtica & Edwards & Corynoneura & Corynoneura (Corynoneura) \\
\hline ceylanicus & Kieffer & Cardiocladius & Cardiocladius \\
\hline ceylonicus (incorr. spellg.) & Kieffer & Cardiocladius & Cardiocladius \\
\hline chalybeata & Edwards & Spaniotoma & Spaniotoma (Trichocladius) \\
\hline cheethami & Edwards & Spaniotoma & Spaniotoma (Smittia) \\
\hline chiron & Haliday & Chironomus & Syndiamesa \\
\hline chloris & Meigen & Chironomus & Chironomus (Microtendipes) \\
\hline chlorolobus & Kieffer & Chironomus & Chironomus (Chironomus) \\
\hline choreus & Meigen & Tanypus & Procladius (Procladius) \\
\hline choreus & Meigen misid. Kieffer & Tanypus & Procladius (Procladius) \\
\hline cincta & Fabricius & Tipula & Tipula \\
\hline cinctellus & Goetghebuer & Chironomus & Chironomus (Chironomus) \\
\hline cinereiventris & Goetghebuer & Chironomus & Chironomus (Microtendipes) \\
\hline cingulata & Walker & Chironomus & Pentaneura \\
\hline cingulatus & Meigen & Chironomus & Chironomus (Chironomus) \\
\hline claripennis & Malloch & Chironomus & Chironomus (Chironomus) \\
\hline clavaticornis & Goetghebuer & Camptocladius & Spaniotoma (Limnophyes) \\
\hline clavaticrus & Kieffer & Chironomus & Chironomus (Lauterborniella) \\
\hline clavicornis & Kieffer & Corynoneura & Corynoneura (Thienemanniella) \\
\hline claviforceps & Edwards & Chironomus & Chironomus (Chironomus) \\
\hline coaequatus & Walker & Chironomus & Spaniotoma (Trichocladius) \\
\hline coerulescens & Kieffer & Trichocladius & Spaniotoma (Eukiefferiella) \\
\hline compertus & Walker & Chironomus & Chironomus \\
\hline confinis & Meigen & Chironomus & Chironomus (Microtendipes) \\
\hline conjuncta & Edwards & Spaniotoma & Spaniotoma (Smittia) \\
\hline consobrinus & Zetterstedt & Tanypus & Prodiamesa \\
\hline contingens & Walker & Chironomus & Spaniotoma (Smittia) \\
\hline convectus & Walker & Chironomus & Chironomus (Chironomus) \\
\hline conversus & Walker & Chironomus & Cricotopus \\
\hline convestitus & Walker & Chironomus & Prodiamesa \\
\hline convictus & Walker & Chironomus & Chironomus (Polypedilum) \\
\hline coracellus & Kieffer & Trichocladius & Chironomus (Endochironomus) \\
\hline coracinum & Zetterstedt & Chironomus & Pentapedilum (Sergentia) \\
\hline coracinus & Zetterstedt misid. Kieffer & Chironomus & Tanytarsus (Lauterbornia) \\
\hline cornutus & Goetghebuer & Tanytarsus & Tanytarsus \\
\hline coronata & Edwards & Corynoneura & Corynoneura (Corynoneura) \\
\hline coronata & Edwards & Spaniotoma & Spaniotoma (Eukiefferiella) \\
\hline costalis & Kieffer & Pelopia & Pentaneura \\
\hline crassinervis & Zetterstedt & Tanypus & Procladius (Procladius) \\
\hline crassipes & Kieffer & Corynoneura & Corynoneura (Corynoneura) \\
\hline crassipes & Panzer & Chironomus & Eurycnemus \\
\hline cubitalis & Kieffer & Metriocnemus & Metriocnemus \\
\hline culiciformis & Linnaeus & Tipula & Procladius (Procladius) \\
\hline culiciformis & Linnaeus & Tipula & Tanypus \\
\hline culicoides & Heeger & Diamesa & Diamesa \\
\hline cultriger & Kieffer & Diplocladius & Spaniotoma (Diplocladius) \\
\hline cuneatus & Edwards & Metriocnemus & Metriocnemus \\
\hline cuneipennis & Edwards & Tanytarsus & Tanytarsus (Stempellina) \\
\hline curticornis & Kieffer & Tanytarsus & Tanytarsus (Tanytarsus) \\
\hline curticosta & Edwards & Spaniotoma & Spaniotoma (Smittia) \\
\hline curtistylus & Goetghebuer, 1921 & Psectrocladius & Spaniotoma (Orthocladius) \\
\hline decipiens & Kieffer & Trichocladius & Trichocladius \\
\hline defectus & Kieffer & Chironomus & Chironomus (Chironomus) \\
\hline denotatus & Walker & Chironomus & Cricotopus \\
\hline denticulatus & Goetghebuer & Chironomus & Chironomus (Chironomus) \\
\hline dentiforceps & Edwards & Spaniotoma & Spaniotoma (Orthocladius) \\
\hline deproperans & Walker & Chironomus & Metriocnemus \\
\hline devonica & Edwards & Spaniotoma & Spaniotoma (Orthocladius) \\
\hline diffinis & Edwards & Chironomus & Chironomus (Microtendipes) \\
\hline digitalis & Edwards & Chironomus & Chironomus (Chironomus) \\
\hline dilatatus & van der Wulp & Chironomus & Spaniotoma (Trichocladius) \\
\hline dilatatus & van der Wulp & Chironomus & Orthocladius \\
\hline
\end{tabular}

312

\section{Page numbers}

368, 369

368, 368f, 429f

317

280

329f, 330k, 331

352f, 359, $428 \mathrm{f}$

304

397, $397 \mathrm{k}$

388

301

301

299

390

397

293, 294

383k, 384

381f, 387

356

405

366

381f, 389

328

280, 351k, 352f, 354

280

397k, 398f, 399, 429f

$363 \mathrm{k}, 365,428 \mathrm{f}$

307

$360 \mathrm{k}, 361$

385

326

307

398f, 401, 402k, 404

394

375, 377, 418

374,418

414

367, 369

$351 \mathrm{k}, 354,368 \mathrm{f}$

290

301

369

309

313

299, 301

300

304

331, 358

315, $428 \mathrm{f}$

421, 429f

$412 \mathrm{k}, 415$

352f, 363k, 364, 428f

335, 350, 359

331

388

326

389

339f, 345k, 346

311

339f, 347k, 349

397, 397k, 398f

381f, 389

328

332 


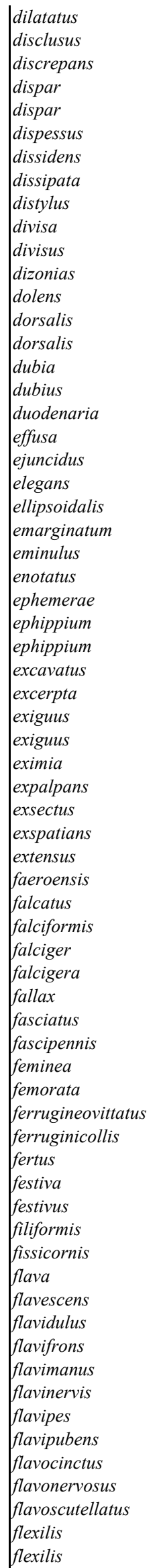

\begin{tabular}{|c|c|}
\hline van der Wulp misid. Goet. & Chironomus \\
\hline Walker & Chironomus \\
\hline Walker & Chironomus \\
\hline Goetghebuer & Cricotopus \\
\hline Meigen & Chironomus \\
\hline Walker & Chironomus \\
\hline Walker & Chironomus \\
\hline Edwards & Spaniotoma \\
\hline Kieffer & Dactylocladius \\
\hline Walker & Chironomus \\
\hline Kieffer & Orthocladius \\
\hline Meigen & Chironomus \\
\hline Walker & Chironomus \\
\hline Meigen & Chironomus \\
\hline Meigen sensu Edw. & Chironomus \\
\hline Meigen & Tanypus \\
\hline Staeger & Tanypus \\
\hline Kieffer & Tvetenia \\
\hline Walker & Chironomus \\
\hline Walker & Chironomus \\
\hline Meigen & Chironomus \\
\hline Kieffer & Orthocladius \\
\hline Kieffer & Polypedilum \\
\hline Walker & Chironomus \\
\hline Walker & Chironomus \\
\hline Kieffer & Camptocladius \\
\hline Zetterstedt & Chironomus \\
\hline Zetterstedt misid. Verrall & Chironomus \\
\hline Edwards & Tanytarsus \\
\hline Walker & Chironomus \\
\hline Goetghebuer & Camptocladius \\
\hline Goetghebuer & Camptocladius \\
\hline Edwards & Pentaneura \\
\hline Walker & Chironomus \\
\hline Kieffer & Tanytarsus \\
\hline Walker & Chironomus \\
\hline Kieffer & Psectrocladius \\
\hline Kieffer & Tendipes \\
\hline Kieffer & Chironomus \\
\hline Kieffer & Tendipes \\
\hline Kieffer & Tendipes \\
\hline Kieffer & Pelopia \\
\hline Kieffer & Trichocladius \\
\hline Geoffroy sensu Kieffer & Tipula \\
\hline Zetterstedt & Chironomus \\
\hline Edwards & Spaniotoma \\
\hline Staeger & Chironomus \\
\hline Zetterstedt & Chironomus \\
\hline Meigen & Tanypus \\
\hline Walker & Chironomus \\
\hline Meigen & Tanypus \\
\hline Meigen & Chironomus \\
\hline Kieffer & Psectrocladius \\
\hline Kieffer & Orthocladius \\
\hline Kieffer & Microlenzia \\
\hline Edwards & Corynoneura \\
\hline Edwards & Tanytarsus \\
\hline Edwards & Procladius \\
\hline Meigen & Chironomus \\
\hline Kieffer & Xenochironom \\
\hline Meigen & Chironomus \\
\hline Goetghebuer & Tanypus \\
\hline Kieffer & Trichocladius \\
\hline Staeger & Chironomus \\
\hline Goetghebuer & Tanypus \\
\hline Linnaeus misid. auct. & Tipula \\
\hline Linnaeus sensu Bause & Tipula \\
\hline
\end{tabular}

\begin{tabular}{|c|c|}
\hline Spaniotoma (Psectrocladius) & 332 \\
\hline Chironomus & 280 \\
\hline Chironomus (Microtendipes) & 397 \\
\hline Spaniotoma (Trichocladius) & $329,329 \mathrm{k}, 330$ \\
\hline Chironomus (Endochironomus) & 374f, 379f, 394 \\
\hline Chironomus (Chironomus) & 386 \\
\hline Chironomus (Chironomus) & 381f, $\mathbf{3 8 5}, 396$ \\
\hline Spaniotoma (Orthocladius) & $337 \mathrm{k}, 338,339 \mathrm{f}, 428 \mathrm{f}$ \\
\hline Orthocladius & 345 \\
\hline Pentaneura & 293,294 \\
\hline Orthocladius & 340 \\
\hline Cricotopus & $320 \mathrm{k}, 321,322 \mathrm{f}$ \\
\hline Chironomus (Chironomus) & 383 \\
\hline Chironomus (Chironomus) & 385 \\
\hline Chironomus & $375,383 \mathrm{k}, 384$ \\
\hline Pentaneura & 295 \\
\hline Pentaneura & 290,291 \\
\hline Tvetenia & 343 \\
\hline Spaniotoma (Trichocladius) & 329f, 330, 330k \\
\hline Tanytarsus (Tanytarsus) & 374f, 414 \\
\hline Eurycnemus & 309 \\
\hline Pachycladius & 430 \\
\hline Polypedilum & 401,402 \\
\hline Tanytarsus (Tanytarsus) & $412 \mathrm{k}, \mathbf{4 1 4}$ \\
\hline Chironomus (Polypedilum) & 402 \\
\hline Spaniotoma (Smittia) & $352 f, 358,428 f, 354$ \\
\hline Cricotopus & 325 \\
\hline Cricotopus & 325 \\
\hline Tanytarsus (Tanytarsus) & 416, $417 f$ \\
\hline Spaniotoma (Orthocladius) & $337 \mathrm{k}, 338,339 \mathrm{f}$ \\
\hline Camptocladius & 280,354 \\
\hline Spaniotoma (Limnophyes) & 355 \\
\hline Pentaneura & $289, \mathbf{2 9 0}, 428 f$ \\
\hline Pentaneura & 293 \\
\hline Tanytarsus (Micropsectra) & 407 \\
\hline Spaniotoma (Psectrocladius) & 334 \\
\hline Spaniotoma (Psectrocladius) & 332 \\
\hline Chironomus (Chironomus) & 387 \\
\hline Chironomus (Chironomus) & 389 \\
\hline Chironomus (Chironomus) & 386 \\
\hline Chironomus (Polypedilum) & 402 \\
\hline Pentaneura & $288,293,294$ \\
\hline Cricotopus & 321 \\
\hline Chironomus (Stenochironomus) & 395 \\
\hline Chironomus (Stenochironomus) & 395 \\
\hline Spaniotoma (Orthocladius) & $339 \mathrm{f}, 340,340 \mathrm{k}, 343,368 \mathrm{f}$ \\
\hline Spaniotoma (Eukiefferiella) & 351 \\
\hline Chironomus (Chironomus) & 384 \\
\hline Pentaneura & 294 \\
\hline Metriocnemus & 311 \\
\hline Pentaneura & 289,291 \\
\hline Cricotopus & $320 \mathrm{k}, 322$ \\
\hline Spaniotoma (Orthocladius) & 350 \\
\hline Trichocladius & 328,335 \\
\hline Corynoneura (Microlenzia) & 367 \\
\hline Corynoneura (Thienemanniella) & $352 \mathrm{f}, \mathbf{3 6 7}, 429 \mathrm{f}$ \\
\hline Tanytarsus (Stempellina) & 420 \\
\hline Procladius (Psilotanypus) & $285 f, 302$ \\
\hline Chironomus & 392 \\
\hline Chironomus (Chironomus) & 385 \\
\hline Pentapedilum (Phaenopsectra) & $374 f, 375$ \\
\hline Anatopynia & 298 \\
\hline Cricotopus & $320 \mathrm{k}, 322 \mathrm{f}, 323$ \\
\hline Chironomus (Polypedilum) & $402 \mathrm{k}, 403$ \\
\hline Pentaneura & 294 \\
\hline Chironomus (Stenochironomus) & 395 \\
\hline Chironomus (Lauterborniella) & 405 \\
\hline
\end{tabular}




\begin{tabular}{|c|c|}
\hline flexuella & Edwards \\
\hline Species-group name & Authorship \\
\hline foenisuga & Kieffer \\
\hline foliacea & Kieffer \\
\hline foliaceus & Kieffer \\
\hline foliatus & Kieffer \\
\hline foliicola & Kieffer \\
\hline forcipatus & Goetghebuer \\
\hline formosus & Goetghebuer \\
\hline foveata & Edwards \\
\hline frauenfeldi & Schiner \\
\hline frigida & Zetterstedt \\
\hline fucicola & Edwards \\
\hline fuliginosus & Goetghebuer \\
\hline fulvofasciatus & Kieffer \\
\hline fulvofasciatus & Kieffer \\
\hline funebris & Goetghebuer \\
\hline furcata & Kieffer \\
\hline fusca & Kieffer \\
\hline fuscicauda & Kieffer \\
\hline fuscicauda & Kieffer \\
\hline fusciceps & Edwards \\
\hline fusciclava & Kieffer \\
\hline fusciforceps & Kieffer \\
\hline fuscihalter & Edwards \\
\hline fuscimanus & Kieffer \\
\hline fuscimanus & Kieffer \\
\hline fuscimanus & Meigen \\
\hline fuscipennis & Meigen \\
\hline fuscipes & Kieffer \\
\hline fuscipes & Meigen \\
\hline fuscipes & Meigen misid. Goet. \\
\hline fuscus & Kieffer \\
\hline fuscus & Meigen \\
\hline fusiformis & Kieffer \\
\hline futilis & Walker \\
\hline gaedii & Meigen \\
\hline galactoptera & Nowicki \\
\hline gibbosus & Meigen \\
\hline gibbus & Fabricius \\
\hline glabrescens & Edwards \\
\hline glabricollis & Meigen \\
\hline glabricollis & Meigen misid. Walker \\
\hline glabripennis & Goetghebuer \\
\hline glauciventris & Kieffer \\
\hline glaucus & Meigen \\
\hline globulifer & Goetghebuer \\
\hline gmundensis & Egger \\
\hline goetghebueri & Kieffer \\
\hline goetghebueri & Kieffer \\
\hline gotchi & Goetghebuer \\
\hline gracei & Edwards \\
\hline gracei & Edwards \\
\hline gracilior & Kieffer \\
\hline gracilis & Goetghebuer \\
\hline gracilis & Kieffer \\
\hline gracilis & Kieffer \\
\hline grandis & Kieffer \\
\hline grandis & Kieffer \\
\hline gregarius & Kieffer \\
\hline grimshawi & Edwards \\
\hline gripekoveni & Kieffer \\
\hline grisea & Philippi \\
\hline griseipennis & van der Wulp \\
\hline gurgicola & Edwards \\
\hline
\end{tabular}

\begin{tabular}{|c|c|}
\hline Spaniotoma & |Spaniotoma (Orthocladius) \\
\hline $\begin{array}{l}\text { Genus in original } \\
\text { combination }\end{array}$ & $\begin{array}{l}\text { Genus-group name(s) } \\
\text { stated or implied by Edwards }\end{array}$ \\
\hline Camptocladius & Spaniotoma (Limnophyes) \\
\hline Camptocladius & Spaniotoma (Smittia) \\
\hline Psectrocladius & Psectrocladius \\
\hline Camptocl., Trichocl. & Spaniotoma (Smittia) \\
\hline Glyptotendipes & Chironoтиs (Glyptotendipes) \\
\hline Camptocladius & Camptocladius \\
\hline Chironomus & Chironomus (Microtendipes) \\
\hline Spaniotoma & Spaniotoma (Trichocladius) \\
\hline Thalassomya & Thalassomyia \\
\hline Chironomus & Spaniotoma (Orthocladius) \\
\hline Trichocladius & Cricotopus \\
\hline Tanypus & Pentaneura \\
\hline Glyptotendipes & Chironomus (Glyptotendipes) \\
\hline Metriocnemus & Metriocnemus \\
\hline Trichocladius & Spaniotoma (Trichocladius) \\
\hline Psectrocladius & Spaniotoma (Orthocladius) \\
\hline Microlenzia & Corynoneura (Thienemanniella) \\
\hline Calopsectra & Phaenopelma \\
\hline Limnochironomus & Chironomus (Chironomus) \\
\hline Pentaneura & Pentaneura \\
\hline Corynoneura & Corynoneura \\
\hline Cricotopus & Cricotopus \\
\hline Corynoneura & Corynoneura (Corynoneura) \\
\hline Harnischia & Harnischia \\
\hline Paratendipes & Chironomus (Paratendipes) \\
\hline Chironomus & Cricotopus \\
\hline Chironomus & Chironomus (Microtendipes) \\
\hline Cricotopus & Spaniotoma (Trichocladius) \\
\hline Chironomus & Metriocnemus \\
\hline Chironomus & Metriocnemus \\
\hline Cardiocladius & Cardiocladius \\
\hline Chironomus & Tanytarsus (Micropsectra) \\
\hline Tendipes & Chironomus \\
\hline Chironomus & Chironomus (Chironomus) \\
\hline Diamesa & Diamesa \\
\hline Diamesa & Diamesa \\
\hline Chironomus & Cricotopus \\
\hline Tipula & Chironomus (Stenochironomus) \\
\hline Tanytarsus & Tanytarsus (Tanytarsus) \\
\hline Chironomus & Spaniotoma (Trichocladius) \\
\hline Chironomus & Chironomus (Chironomus) \\
\hline Dactylocladius & Spaniotoma (Orthocladius) \\
\hline Trichocladius & Cricotopus \\
\hline Chironomus & Chironomиs (Glyptotendipes) \\
\hline Tanytarsus & Tanytarsus \\
\hline Chironomus & Tanytarsus (Micropsectra) \\
\hline Chironomus & Chironomus (Chironomus) \\
\hline Tanypus & Anatopynia \\
\hline Tanytarsus & Tanytarsus (Tanytarsus) \\
\hline Metriocnemus & Metriocnemus \\
\hline Spaniotoma & Spaniotoma (Orthocladius) \\
\hline Chironomus & Chironomus (Chironomus) \\
\hline Camptocladius & Spaniotoma (Smittia) \\
\hline Syndiamesa & Syndiamesa (Lasiodiamesa) \\
\hline Thienemannia & Metriocnemus \\
\hline Acricotopus & Acricotopus \\
\hline Acricotopus & Spaniotoma (Trichocladius) \\
\hline Tanytarsus & Tanytarsus (Tanytarsus) \\
\hline Metriocnemus & Metriocnemus \\
\hline Chironomus & Chironoтиs (Glyptotendipes) \\
\hline Pentaneura & Pentaneura \\
\hline Tanypus & Pentaneura \\
\hline Spaniotoma & Spaniotoma (Limnophyes) \\
\hline
\end{tabular}

339f, 349, $428 \mathrm{f}$

Page numbers

355

360k, 361

362

360

391k, 392, $398 \mathrm{f}$

365

397

329f, 330, 330k, $428 \mathrm{f}$

$371,429 \mathrm{f}$

339f, 345k, 346

$324 \mathrm{k}, 326$

293

393

313

328,360

281, 340k, 341

367

419

386

289, 290, $306 f$

369

319

369, $429 f$

387

396

319, $319 \mathrm{k}$

396, 397k, 400

330

281, 310, 311, 311k

311

280, 317

407

373

386

307

307

321

379f, 395, 399, 405

416, $417 \mathrm{f}$

329f, 330, 330k

390

344k, 345

325

379f, 391k, 392

407

407

386

298

$412 \mathrm{k}, 414$

$311 \mathrm{k}, 312,314 \mathrm{f}$

339f, 345k, 346, 428f

389

362

296

310,313

328

328

411, $412 \mathrm{k}, 414$

$313,428 \mathrm{f}$

391k, 392

288

292, 293

$355 \mathrm{k}, 357,428 \mathrm{f}$ 


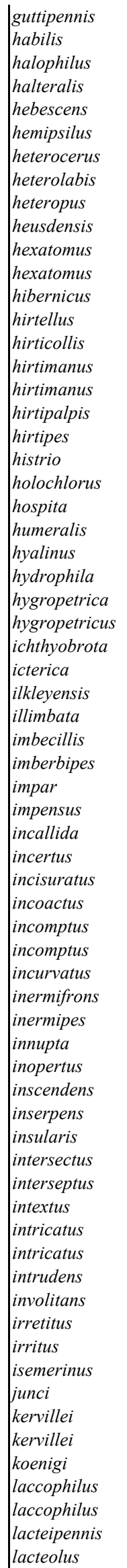

\begin{tabular}{|c|c|c|}
\hline van der Wulp & Tanypus & Pentaneura \\
\hline Walker & Chironomus & Spaniotoma (Limnophyes) \\
\hline Kieffer & Trichocladius & Trichocladius \\
\hline Coquillett & Chironomus & Chironomus \\
\hline Walker & Chironomus & Chironomus (Chironomus) \\
\hline Kieffer & Tanytarsus & Tanytarsus (Micropsectra) \\
\hline Kieffer & Trissocladius & Trissocladius \\
\hline Kieffer & Chironomus & Chironomus (Chironomus) \\
\hline Kieffer & Glyptotendipes & Chironomus (Glyptotendipes) \\
\hline Goetghebuer & Tanytarsus & Tanytarsus \\
\hline Kieffer & Camptocladius & Camptocladius \\
\hline Kieffer & Camptocladius & Spaniotoma (Limnophyes) \\
\hline Edwards & Chironomus & Chironomus (Microtendipes) \\
\hline Goetghebuer & Metriocnemus & Metriocnemus \\
\hline Staeger & Chironomus & Metriocnemus \\
\hline Kieffer & Chironomus & Chironomus (Chironomus) \\
\hline Kieffer & Tanypus & Pentaneura \\
\hline Kieffer & Metriocnemus & Metriocnemus \\
\hline Macquart & Chironomus & Eurycnemus \\
\hline Fabricius & Tipula & Chironomus (Stictochironomus) \\
\hline Edwards & Tanytarsus & Tanytarsus (Tanytarsus) \\
\hline Edwards & Spaniotoma & Spaniotoma (Eukiefferiella) \\
\hline Zetterstedt & Ceratopogon & Cricotopus \\
\hline Kieffer & Cricotopus & Cricotopus \\
\hline Goetghebuer & Camptocladius & Spaniotoma (Limnophyes) \\
\hline Kieffer & Diamesa & Diamesa \\
\hline Kieffer & Metriocnemus & Metriocnemus \\
\hline Kieffer & Prodiamesa & Prodiamesa \\
\hline Meigen & Chironomus & Spaniotoma (Orthocladius) \\
\hline Edwards & Spaniotoma & Spaniotoma (Orthocladius) \\
\hline Edwards & Spaniotoma & Spaniotoma (Orthocladius) \\
\hline Walker & Chironomus & Chironomus (Glyptotendipes) \\
\hline Kieffer & Chironomus & Chironomus (Chironomus) \\
\hline Walker & Chironomus & Chironomus (Endochironomus) \\
\hline Walker & Chironomus & Metriocnemus \\
\hline Walker & Chironomus & Diamesa \\
\hline Walker & Chironomus & Chironomus (Chironomus) \\
\hline Zetterstedt & Chironomus & Cricotopus \\
\hline Walker & Chironomus & Spaniotoma (Orthocladius) \\
\hline Walker & Chironomus & Procladius (Procladius) \\
\hline Zetterstedt & Chironomus & Pentapedilum (Phaenopsectra) \\
\hline Goetghebuer & Tanypus & Pentaneura \\
\hline Goetghebuer & Chironomus & Chironomus (Chironomus) \\
\hline Kieffer & Tanytarsus & Micropsectra \\
\hline Edwards & Corynoneura & Corynoneura (Corynoneura) \\
\hline Walker & Chironomus & Tanytarsus (Tanytarsus) \\
\hline Walker & Chironomus & Diamesa \\
\hline Walker & Chironomus & Cricotopus \\
\hline Kieffer & Tanytarsus & Tanytarsus (Micropsectra) \\
\hline Staeger & Chironomus & Cricotopus \\
\hline Walker & Chironomus & Pentaneura \\
\hline Walker & Chironomus & Chironomus (Endochironomus) \\
\hline Goetghebuer & Tanytarsus & Tanytarsus (Phaenopelma) \\
\hline Goetghebuer misid. Edw. & Tanytarsus & Tanytarsus \\
\hline Walker & Chironomus & Tanytarsus (Micropsectra) \\
\hline Walker & Chironomus & Chironomus (Endochironomus) \\
\hline Walker & Chironomus & Chironomus (Chironomus) \\
\hline Walker & Chironomus & Metriocnemus \\
\hline Chevrel & Scopelodromus & Thalassomyia \\
\hline Meigen & Chironomus & Tanytarsus (Tanytarsus) \\
\hline Kieffer & Orthocladius & Cricotopus \\
\hline Kieffer & Orthocladius & Orthocladius \\
\hline Kieffer & Chironomus & Tanytarsus (Lauterbornia) \\
\hline Edwards & Tanytarsus & Tanytarsus (Lundstroemia) \\
\hline Kieffer & Trichocladius & Cricotopus \\
\hline Zetterstedt & Tanypus & Diamesa \\
\hline Goetghebuer & Metriocnemus & Metriocnemus \\
\hline
\end{tabular}

288, 289

$355 \mathrm{k}, 356$

318,326

403

384

407

309

386

392

416

354

355

396, 397k, 398f, 399

312

$311 \mathrm{k}, 312$

384

290, 291

311

309

401

412k, 414

351, 351k, 352f, 368f, 428f

325

319

$355,355 \mathrm{k}$

303

311

307

340, 340k

339f, 347k, 349

339f, 340, 340k, 343, $428 \mathrm{f}$

$391 \mathrm{k}, 393$

388

394, $429 f$

314

305, $306 f$

389

323

345

301

375

291

$383 \mathrm{k}, 384$

407

368f, 369

410, $412 \mathrm{k}$

306, $306 \mathrm{f}$

322f, 324k, 326

407

323, $324 \mathrm{k}$

293

394

419

411

407

394

389

314f, 315

371

415

325

335

$374,377,418$

409, $412 \mathrm{k}$

322

307

312 


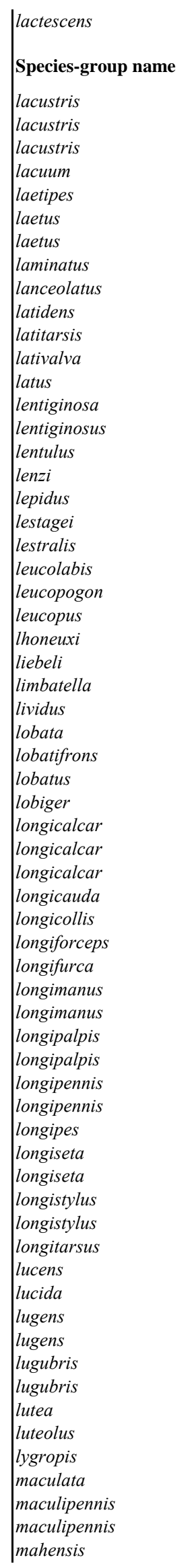

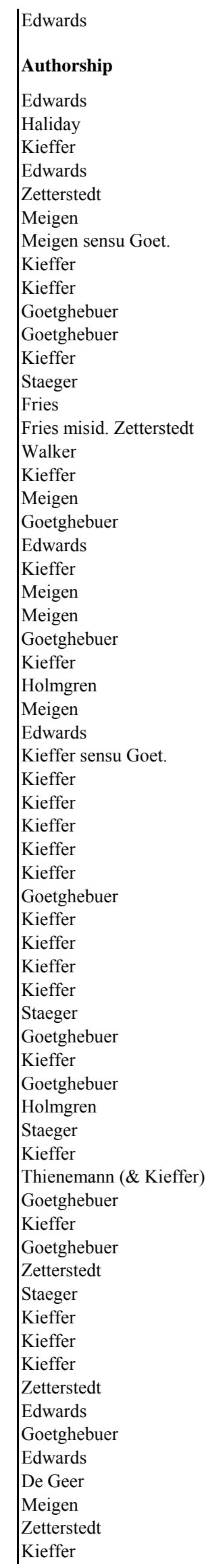

\begin{tabular}{|c|c|}
\hline Tanytarsus & Tanytarsus (Tanytarsus) \\
\hline $\begin{array}{l}\text { Genus in original } \\
\text { combination }\end{array}$ & $\begin{array}{l}\text { Genus-group name(s) } \\
\text { stated or implied by Edwards }\end{array}$ \\
\hline Corynoneura & Corynoneura (Corynoneura) \\
\hline Chironomus & Chironomus (Chironomus) \\
\hline Cricotopus & Cricotopus \\
\hline Cricotopus & Cricotopus \\
\hline Chironomus & Tanytarsus (Tanytarsus) \\
\hline Chironomus & Chironomus (Polypedilum) \\
\hline Tanypus & Pentaneura \\
\hline Cladopelma & Chironomus (Chironomus) \\
\hline Tanytarsus & Tanytarsus (Micropsectra) \\
\hline Chironomus & Chironomus (Chironomus) \\
\hline Psilodiamesa & Diamesa \\
\hline Chironomus & Chironomus \\
\hline Chironomus & Chironomus \\
\hline Tanypus & Pentaneura \\
\hline Tanypus & Pentaneura \\
\hline Chironomus & Chironomus (Microtendipes) \\
\hline Orthocladius & Spaniotoma (Orthocladius) \\
\hline Chironomus & Chironomus (Endochironomus) \\
\hline Tanytarsus & Tanytarsus (Tanytarsus) \\
\hline Trichocladius & Cricotopus \\
\hline Chironomus & Pentapedilum (Phaenopsectra) \\
\hline Chironomus & Spaniotoma (Smittia) \\
\hline Chironomus & Chironomus (Polypedilum) \\
\hline Chironomus & Chironomus (Chironomus) \\
\hline Tendipes & Chironomus (Chironomus) \\
\hline Chironomus & Spaniotoma (Psectrocladius) \\
\hline Chironomus & Chironomus (Microtendipes) \\
\hline Corynoneura & Corynoneura (Corynoneura) \\
\hline Tanytarsus & Tanytarsus (Tanytarsus) \\
\hline Trichocladius & Spaniotoma (Trichocladius) \\
\hline Limnochironomus & Chironomus (Chironomus) \\
\hline Dactylocladius & Dactylocladius \\
\hline Psectrotanypus & Psectrotanypus \\
\hline Psectrotanypus & Anatopynia \\
\hline Chironomus & Chironomus (Chironomus) \\
\hline Metriocnemus & Metriocnemus \\
\hline Chironomus & Chironomus (Chironomus) \\
\hline Brillia & Brillia \\
\hline Tanytarsus & Tanytarsus (Micropsectra) \\
\hline Tanypus & Pentaneura \\
\hline Tanypus & Pentaneura \\
\hline Cricotopus & Cricotopus \\
\hline Psectrotanypus & Pentaneura \\
\hline Smittia & Metriocnemus \\
\hline Chironomus & Chironomus (Chironomus) \\
\hline Calopsectra & Pentapedilum (Pentapedilum) \\
\hline Camptocladius & Spaniotoma (Limnophyes) \\
\hline Chironomus & Chironomus (Chironomus) \\
\hline Camptocladius & Pachycladius \\
\hline Metriocnemus & Metriocnemus \\
\hline Chironomus & Spaniotoma (Trichocladius) \\
\hline Chironomus & Spaniotoma (Trichocladius) \\
\hline Procladius & Procladius (Psilotanypus) \\
\hline Tanytarsus & Tanytarsus (Tanytarsus) \\
\hline Microtendipes & Chironomus (Microtendipes) \\
\hline Chironomus & Chironomus (Chironomus) \\
\hline Corynoneura & Corynoneura (Thienemanniella) \\
\hline Psectrocladius & Spaniotoma (Psectrocladius) \\
\hline Cricotopus & Cricotopus \\
\hline Tipula & Tipula \\
\hline Chironomus & Chironomus (Stictochironomus) \\
\hline Tanypus & Pentaneura \\
\hline Metriocnemus & Metriocnemus \\
\hline
\end{tabular}

416, $417 f$

Page numbers

368

390,391

323

$322 \mathrm{f}, 324,324 \mathrm{k}$

407, 410, 416

401k, 402

290

381f, 387

407

385

305, $306 f$

375

376

289, 290

290

399

345

394

415

325

375

360k, 361

401k, 403

388

383

332k, 334

397

368

411

328

381f, 386

350

297

299

390

313

388

310, 314f, $428 \mathrm{f}$ 407

292, 293

294

319

293

357

385

376

355

383k, 384

430

311, $311 \mathrm{k}$

328

$325, \mathbf{3 2 8}, 332,360$

302

411, 414

397

$383,383 \mathrm{k}$

366, $429 f$

334

$324 \mathrm{k}, 325$

299

400

289, 290

316 


\begin{tabular}{|c|c|}
\hline majuscula & Edwards \\
\hline malacus & Walker \\
\hline mancunianus & Edwards \\
\hline mancus & Walker \\
\hline marcidus & Walker \\
\hline marginatus & Macquart \\
\hline marinus & Haliday \\
\hline maritimus & Kieffer \\
\hline marmorata & van der Wulp \\
\hline martinii & Thienemann \\
\hline $\operatorname{maxi}$ & Goetghebuer \\
\hline melaleuca & Meigen \\
\hline melanops & Meigen \\
\hline melanurus & Meigen \\
\hline migrator & Kieffer \\
\hline miki & Kieffer \\
\hline minima & Meigen \\
\hline minimus & Meigen \\
\hline minor & Edwards \\
\hline minor & Edwards \\
\hline minusculus & Edwards \\
\hline minusculus & Walker \\
\hline minuta & Winnertz \\
\hline miriforceps & Kieffer \\
\hline modesta & Meigen \\
\hline modicellus & Walker \\
\hline moerens & Walker \\
\hline monilis & Linnaeus \\
\hline monilis & Linnaeus \\
\hline monochromus & van der Wulp \\
\hline monotomus & Kieffer \\
\hline monticola & Edwards \\
\hline montium & Edwards \\
\hline montivagus & Goetghebuer \\
\hline morio & Zetterstedt \\
\hline morosa & Edwards \\
\hline morulus & Walker \\
\hline motitator & Linnaeus sensu Meigen \\
\hline motitatrix & Linnaeus \\
\hline moturus & Walker \\
\hline mucronatus & Goetghebuer \\
\hline murinus & Goetghebuer \\
\hline muscicola & Kieffer \\
\hline muscicola & Kieffer \\
\hline nactus & Walker \\
\hline nanulus & van der Wulp \\
\hline nebulosa & Meigen \\
\hline nemoralis & Zetterstedt \\
\hline nemorosus & Edwards \\
\hline nemorum & Goetghebuer \\
\hline nervosus & Meigen \\
\hline nervosus & Staeger \\
\hline nexilis & Walker \\
\hline nidorum & Edwards \\
\hline niger & Meigen \\
\hline nigerrima & Goetghebuer \\
\hline nigratus & Walker \\
\hline nigricans & Goetghebuer \\
\hline nigrimanus & Staeger \\
\hline nigrimanus & Staeger misid. auct. \\
\hline nigritulus & Goetghebuer \\
\hline nigriventris & van der Wulp \\
\hline nigrofasciatus & Goetghebuer \\
\hline nigronitans (var. or. spellg.) & Edwards \\
\hline nigronitens & Edwards \\
\hline nigropunctata & Staeger \\
\hline nigrovittatus & Goetghebuer \\
\hline
\end{tabular}

\begin{tabular}{|c|c|}
\hline Corynoneura & |Corynoneura (Thienemanniella) \\
\hline Chironomus & Chironomus (Chironomus) \\
\hline Chironomus & Chironomus (Glyptotendipes) \\
\hline Chironomus & Tanytarsus (Tanytarsus) \\
\hline Chironomus & Metriocnemus \\
\hline Chironomus & Cricotopus \\
\hline Clunio & Clunio \\
\hline Endochrionomus & Chironomus (Endochironomus) \\
\hline Chironomus & Chironomus (Lauterborniella) \\
\hline Metriocnemus & Metriocnemus \\
\hline Tanypus & Pentaneura \\
\hline Chironomus & Spaniotoma (Orthocladius) \\
\hline Tanypus & Pentaneura \\
\hline Tanypus & Pentaneura \\
\hline Psectrotanypus & Psectrotanypus \\
\hline Chironomus & Chironomus (Endochironomus) \\
\hline Chironomus & Spaniotoma (Limnophyes) \\
\hline Chironomus & Camptocladius \\
\hline Spaniotoma & Spaniotoma (Orthocladius) \\
\hline Tanytarsus & Tanytarsus (Stempellina) \\
\hline Tanytarsus & Tanytarsus (Stempellina) \\
\hline Chironomus & Chironomus \\
\hline Corynoneura & Corynoneura (Corynoneura) \\
\hline Limnochironomus & Chironomus (Chironomus) \\
\hline Chironomus & Brillia \\
\hline Chironomus & Tanytarsus (Tanytarsus) \\
\hline Chironomus & Chironomus (Chironomus) \\
\hline Tipula & Pentaneura \\
\hline Tipula & Tipula \\
\hline Chironomus & Chironomus (Chironomus) \\
\hline Cryptochironomus & Chironomus (Chironomus) \\
\hline Tanytarsus & Tanytarsus (Micropsectra) \\
\hline Diamesa & Diamesa \\
\hline Trichocladius & Cricotopus \\
\hline Tanypus & Protanypus \\
\hline Corynoneura & Corynoneura (Thienemanniella) \\
\hline Chironomus & Chironomus \\
\hline Tipula & Cricotopus \\
\hline Tipula & Tipula \\
\hline Chironomus & Chironomus \\
\hline Chironomus & Chironomus (Chironomus) \\
\hline Tanypus & Pentaneura \\
\hline Orthocladius & Spaniotoma (Orthocladius) \\
\hline Pelopia & Pentaneura \\
\hline Chironomus & Tanytarsus (Micropsectra) \\
\hline Orthocladius & Orthocladius \\
\hline Tanypus & Anatopynia \\
\hline Chironomus & Chironomus (Chironomus) \\
\hline Tanytarsus & Tanytarsus (Tanytarsus) \\
\hline Tanypus & Pentaneura \\
\hline Tanypus & Clinotanypus \\
\hline Chironomus & Chironomus (Chironomus) \\
\hline Chironomus & Diamesa \\
\hline Spaniotoma & Spaniotoma (Orthocladius) \\
\hline Chironomus & Chironomus (Chironomus) \\
\hline Trissocladius & Spaniotoma (Trissocladius) \\
\hline Chironomus & Spaniotoma (Smittia) \\
\hline Tanypus & Pentaneura \\
\hline Chironomus & Chironomus (Chironomus) \\
\hline Chironomus & Chironomus (Chironomus) \\
\hline Zavrelia & Tanytarsus (Zavrelia) \\
\hline Chironomus & Cricotopus \\
\hline Tanytarsus & Tanytarsus \\
\hline Chironomus & Chironomus (Chironomus) \\
\hline Chironomus & Chironomus (Chironomus) \\
\hline Tanypus & Pentaneura \\
\hline Tanytarsus & Tanytarsus (Tanytarsus) \\
\hline
\end{tabular}

366

388

391k, 393, $398 \mathrm{f}$

418

313

319

371, $429 f$

394

380, 405

281, 311k, 312

293

$337,337 \mathrm{k}$

292, 293, 294

292, 293

293

394

355, 355k, $368 \mathrm{f}$

357

339f, 347k, 348, 368f, 428f

420

$417 \mathrm{f}$

280

367,368

386

310

418

383

289, 290

299, 405

389

390

408, $412 \mathrm{k}$

306f, 307, $428 \mathrm{f}$

321

303

365,367

280

$320 \mathrm{k}, 323$

405

280

389

293

340,342

292

407

351

298

384

416, $417 \mathrm{f}$

293, 294

302

381f, 386

305

339f, 340k, 342

383

346

360

293, 294

390

386

419

326

407, 411

380

381f, 390

292, 293, 294

418 


\begin{tabular}{|c|c|}
\hline nitidicollis & Goetghebuer \\
\hline Species-group name & Authorship \\
\hline nitidicollis & Walker \\
\hline nitidus & Kieffer \\
\hline nitidus & Malloch \\
\hline nitidus & Meigen \\
\hline niveipennis & Fabricius \\
\hline northumbrica & Edwards \\
\hline notabilis & Macquart \\
\hline notabilis & Skuse \\
\hline notata & Meigen \\
\hline notata & Staeger \\
\hline notatus & Meigen \\
\hline notescens & Walker \\
\hline novatus & Walker \\
\hline nubeculosus & Meigen \\
\hline nubens & Edwards \\
\hline nubila & Meigen \\
\hline nubilipennis & Meigen \\
\hline nubilus & Meigen misid. Verrall \\
\hline nudipennis & Goetghebuer \\
\hline nudipes & Goetghebuer \\
\hline nudipes & Kieffer \\
\hline nudipes & Zetterstedt \\
\hline nudisquama & Edwards \\
\hline nugax & Walker \\
\hline nympha & Kieffer \\
\hline obditus & Walker \\
\hline objectans & Walker \\
\hline oblidens & Walker \\
\hline obnixus & Walker \\
\hline obreptans & Walker \\
\hline obscurimanus & Zetterstedt \\
\hline obscuripes & Meigen \\
\hline obsepiens & Walker \\
\hline obsistens & Walker \\
\hline obtexens & Walker \\
\hline obtusidens & Goetghebuer \\
\hline obvertens & Walker \\
\hline obvia & Walker \\
\hline occipiens & Walker \\
\hline occultus & Kieffer \\
\hline ochraceus & Goetghebuer \\
\hline offectus & Walker \\
\hline olivacea & Meigen \\
\hline olivaceus & Goetghebuer \\
\hline opacus & Meigen \\
\hline oppertus & Walker \\
\hline oppetitus & Walker \\
\hline opplens & Walker \\
\hline ornata & Meigen \\
\hline ornaticollis & Edwards \\
\hline ornatus & Meigen \\
\hline orophilus & Edwards \\
\hline oscillator & Meigen \\
\hline paganicus & Walker \\
\hline paganus & Meigen \\
\hline pallens & Meigen \\
\hline pallens & Meigen misid. Goet. \\
\hline pallidicornis & Walker \\
\hline pallidipes & Edwards \\
\hline pallidivittatus & Malloch \\
\hline pallidula & Meigen \\
\hline paludicola & Skuse \\
\hline
\end{tabular}

\begin{tabular}{|c|c|}
\hline Camptocladius & Spaniotoma (Orthocladius) \\
\hline $\begin{array}{l}\text { Genus in original } \\
\text { combination }\end{array}$ & $\begin{array}{l}\text { Genus-group name(s) } \\
\text { stated or implied by Edwards }\end{array}$ \\
\hline Chironomus & Spaniotoma (Trichocladius) \\
\hline Cardiocladius & Cardiocladius \\
\hline Trichocladius & Trichocladius \\
\hline Chironomus & Chironomus (Microtendipes) \\
\hline Chironomus & Chironomus (Chironomus) \\
\hline Pentaneura & Pentaneura \\
\hline Chironomus & Chironomus (Endochironomus) \\
\hline Isoplastus & Isoplastus \\
\hline Tanypus & Anatopynia \\
\hline Diamesa & Prodiamesa \\
\hline Chironomиs & Chironomus (Chironomus) \\
\hline Chironomus & Tanytarsus (Micropsectra) \\
\hline Chironomus & Spaniotoma (Orthocladius) \\
\hline Chironomus & Chironomus (Polypedilum) \\
\hline Pentapedilum & Pentapedilum (Pentapedilum) \\
\hline Tanypus & Pentaneura \\
\hline Chironomus & Chironomus (Polypedilum) \\
\hline Chironomиs & Chironomus (Polypedilum) \\
\hline Camptocladius & Spaniotoma (Smittia) \\
\hline Trichocladius & Spaniotoma (Trichocladius) \\
\hline Tanypus & Pentaneura \\
\hline Tanypus & Prodiamesa \\
\hline Chironomus & Chironomus (Paratendipes) \\
\hline Chironomus & Anatopynia \\
\hline Polypedilum & Chironomus (Polypedilum) \\
\hline Chironomus & Prodiamesa \\
\hline Chironomus & Chironomus (Chironomus) \\
\hline Chironomus & Spaniotoma (Orthocladius) \\
\hline Chironomus & Cricotopus \\
\hline Chironomus & Chironomus (Chironomus) \\
\hline Chironomus & Cricotopus \\
\hline Chironomus & Chironomus (Glyptotendipes) \\
\hline Chironomus & Spaniotoma (Trichocladius) \\
\hline Chironomus & Metriocnemus \\
\hline Chironomus & Cricotopus \\
\hline Chironomus & Chironomus (Chironomus) \\
\hline Chironomus & Chironomus (Microtendipes) \\
\hline Chironomus & Spaniotoma (Psectrocladius) \\
\hline Chironomus & Tanytarsus (Micropsectra) \\
\hline Stenochironoтиs & Chironomus (Stenochironomus) \\
\hline Dactylocladius & Spaniotoma (Orthocladius) \\
\hline Chironomus & Tanytarsus (Micropsectra) \\
\hline Chironomus & Prodiamesa \\
\hline Tanytarsus & Tanytarsus (Tanytarsus) \\
\hline Chironomus & Spaniotoma (Smittia) \\
\hline Chironomus & Spaniotoma (Psectrocladius) \\
\hline Chironomus & Chironomus (Stictochironomus) \\
\hline Chironomus & Spaniotoma (Trichocladius) \\
\hline Tanypus & Pentaneura \\
\hline Spaniotoma & Spaniotoma (Smittia) \\
\hline Chironomus & Cricotopus \\
\hline Chironomиs & Chironomus (Lauterborniella) \\
\hline Chironomus & Cricotopus \\
\hline Chironomus & Metriocnemus \\
\hline Chironomus & Chironomus (Chironomus) \\
\hline Chironomus & Chironomus (Glyptotendipes) \\
\hline Chironomus & Chironomus (Glyptotendipes) \\
\hline Chironomus & Tanytarsus (Tanytarsus) \\
\hline Cricotopus & Cricotopus \\
\hline Chironomus & Chironomus (Chironomus) \\
\hline Tanypus & Pentaneura \\
\hline Procladius & Procladius \\
\hline
\end{tabular}

340, 340k, 343

Page numbers

328

317

331

396, 397k, 400

383,393

290, 291, $428 \mathrm{f}$

394

299

298

307

386

407

337

401, 401k, 402,

$402 \mathrm{k}, 403,404$

376, 429f

290, 291, 293

402

402

360k, 362

329

291

307

396, $398 \mathrm{f}$

298

404

307

386

335, 339f, 344k, 345

$322 \mathrm{f}, 324 \mathrm{k}, 326$

388

319

392

328

311

$322 \mathrm{f}, 324 \mathrm{k}, 326$

$383 \mathrm{k}, 384$

399

332, $332 \mathrm{k}$

407

395

340

407

307, $428 f$

418

360

332

401

329

289, 290

352f, 359

319, 319k

398f, 405, 429f

319

311

385, 391

392

392

415

322f, 324, 324k

381f, 382

290, 292

300 


\begin{tabular}{|c|c|}
\hline parilis & Walker \\
\hline paripes & Edwards \\
\hline patens & Walker \\
\hline patibilis & Walker \\
\hline pectinata & Deby \\
\hline pectoralis & Kieffer \\
\hline pedellus & De Geer \\
\hline pedestris & Meigen \\
\hline pedestris & Wollaston nec Meigen \\
\hline penerasus & Edwards \\
\hline penicillatus & Goetghebuer \\
\hline pentachaetus & Kieffer \\
\hline pentaplastus & Kieffer \\
\hline pentastictus & Kieffer \\
\hline peregrinus & Edwards \\
\hline perennis & Meigen \\
\hline perexilis & Walker \\
\hline pergens & Walker \\
\hline perlevis & Walker \\
\hline permacer & Walker \\
\hline perniger & Zetterstedt \\
\hline perpessus & Walker \\
\hline perpulcher & Mitchell \\
\hline perreptans & Walker \\
\hline persidens & Walker \\
\hline pertenuis & Walker \\
\hline pertractus & Walker \\
\hline pervulsus & Walker \\
\hline petrensis & Kieffer \\
\hline phatta & Egger \\
\hline photophilus & Goetghebuer \\
\hline picipes & Meigen \\
\hline pictimanus & Kieffer \\
\hline pictipennis & Skuse \\
\hline pictipes & Zetterstedt \\
\hline pictulus & Meigen \\
\hline piger & Goetghebuer \\
\hline pigra & Goetghebuer \\
\hline pilicornis & Fabricius \\
\hline pilitarsis & Zetterstedt \\
\hline pilosa & Kieffer \\
\hline platypus & Edwards \\
\hline plebeius & Meigen \\
\hline ploenensis & Kieffer \\
\hline plumipes & Fries \\
\hline plumipes & Fries misid. auct. \\
\hline plumosus & Linnaeus \\
\hline polychaetus & Kieffer \\
\hline posticalis & Lundbeck \\
\hline praecox & Kieffer \\
\hline praecox & Meigen \\
\hline prasinatus & Staeger \\
\hline prasinus & Meigen \\
\hline praticola & Kieffer \\
\hline pratorum & Goetghebuer \\
\hline profundorum & Kieffer \\
\hline prolixitarsis & Lundström \\
\hline prolongata & Kieffer \\
\hline prolongata & Kieffer \\
\hline proximus & Kieffer \\
\hline pseudopedellus & Goetghebuer \\
\hline pseudornatus & Goetghebuer \\
\hline pseudosimplex & Goetghebuer \\
\hline pseudotenellulus & Goetghebuer \\
\hline pseudotener & Goetghebuer \\
\hline
\end{tabular}

\begin{tabular}{|c|c|}
\hline Chironomus & |Chironomus (Chironomus) \\
\hline Chironomus & Chironomus (Glyptotendipes) \\
\hline Chironomus & Chironomus (Microtendipes) \\
\hline Chironomus & Spaniotoma (Trichocladius) \\
\hline Psamathiomya & Psammathiomyia \\
\hline Einfeldia & Einfeldia \\
\hline Tipula & Chironomus (Microtendipes) \\
\hline Chironomus & Chironomus (Microtendipes) \\
\hline Chironomиs & Thalassomyia \\
\hline Metriocnemus & Metriocnemus \\
\hline Tanytarsus & Tanytarsus (Lundstroemia) \\
\hline Dactylocladius & Spaniotoma (Orthocladius) \\
\hline Camptocladius & Spaniotoma (Limnophyes) \\
\hline Procladius & Procladius \\
\hline Podonomus & Podonomus \\
\hline Chironomus & Spaniotoma (Orthocladius) \\
\hline Chironomus & Spaniotoma (Eukiefferiella) \\
\hline Chironomus & Diamesa \\
\hline Chironomus & Tanytarsus (Tanytarsus) \\
\hline Chironomus & Diamesa \\
\hline Chironomus & Spaniotoma (Trichocladius) \\
\hline Chironomus & Prodiamesa \\
\hline Chironomиs & Chironomus \\
\hline Chironomus & Chironomus (Microtendipes) \\
\hline Chironomus & Spaniotoma (Orthocladius) \\
\hline Chironomus & Spaniotoma (Orthocladius) \\
\hline Chironomus & Diamesa \\
\hline Chironomus & Spaniotoma (Trichocladius) \\
\hline Brillia & Brillia \\
\hline Tanypus & Pentaneura \\
\hline Tanytarsus & Tanytarsus (Tanytarsus) \\
\hline Chironomus & Metriocnemus \\
\hline Trichocladius & Cricotopus \\
\hline Procladius & Procladius \\
\hline Chironomus & Chironomus (Microtendipes) \\
\hline Chironomus & Chironomus (Stictochironomus) \\
\hline Dactylocladius & Spaniotoma (Orthocladius) \\
\hline Dactylocladius & Spaniotoma (Orthocladius) \\
\hline Tipula & Chironomus (Chironomus) \\
\hline Chironomus & Cricotopus \\
\hline Syndiamesa & Syndiamesa \\
\hline Spaniotoma & Spaniotoma (Psectrocladius) \\
\hline Chironomus & Chironomus (Paratendipes) \\
\hline Proriethia & Pseudochironomus \\
\hline Tanypus & Tanypus \\
\hline Tanypus & Anatopynia \\
\hline Tipula & Chironomus (Chironomus) \\
\hline Dactylocladius & Spaniotoma (Orthocladius) \\
\hline Tanypus & Trichotanypus \\
\hline Diamesa & Prodiamesa \\
\hline Chironomus & Tanytarsus (Lundstroemia) \\
\hline Chironomus & Pseudochironoтиs \\
\hline Chironomus & Chironomus (Chironomus) \\
\hline Tanytarsus & Tanytarsus \\
\hline Camptocladius & Spaniotoma (Smittia) \\
\hline Sergentia & Pentapedilum (Sergentia) \\
\hline Chironomиs & Chironomus (Polypedilum) \\
\hline Camptocladius & Spaniotoma (Limnophyes) \\
\hline Diamesa & Diamesa \\
\hline Trichocladius & Spaniotoma (Trichocladius) \\
\hline Chironomus & Chironomus (Microtendipes) \\
\hline Tanypus & Pentaneura \\
\hline Chironomus & Chironomus (Chironomus) \\
\hline Tanytarsus & Tanytarsus (Lundstroemia) \\
\hline Cryptochirono & Chironomus (Chironomus) \\
\hline
\end{tabular}

389

391k, 392, 429f

400

328

370f, 371, 429f

385

281, 374f, 379f,

397, 397k, 398f

$371,400,401$

371

314f, 315, $428 \mathrm{f}$

374f, 409

337

356

300

280, 296, 306f, $428 \mathrm{f}$

$331,336,337,337 \mathrm{k}, 339 \mathrm{f}$

351

304

410

305, $306 f$

329

307

405

399

345

340

304

328

310

288, 289

412k, 413

311, 311k

320

300

400

379f, 400

$338,339 \mathrm{f}$

$337,337 \mathrm{k}$

298, 377, 383, 383k, 393

319, 319k

303, $428 \mathrm{f}$

$332 \mathrm{k}, 333,428 \mathrm{f}$

396

377

297

298

281, 374f, 382 ,

$383 \mathrm{k}, \mathbf{3 8 4}, 392$

337

296, 300

307

374f, 408

374f, 377, $429 \mathrm{f}$

384

408

360k, 361

374,375

398f, 402k, 403

$355 \mathrm{k}, 356$

305, $306 \mathrm{f}$

329

399

291

390

409

389 


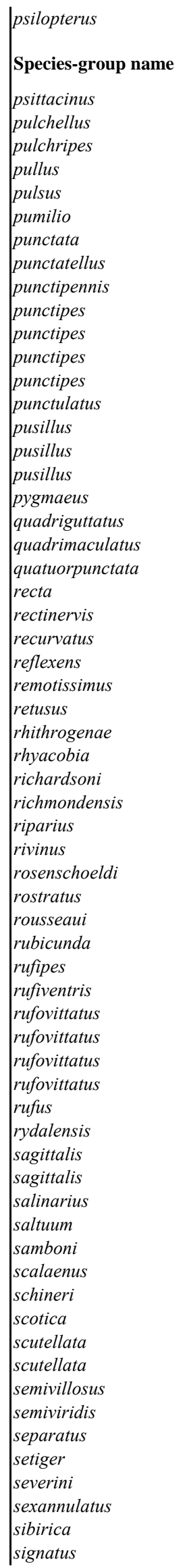

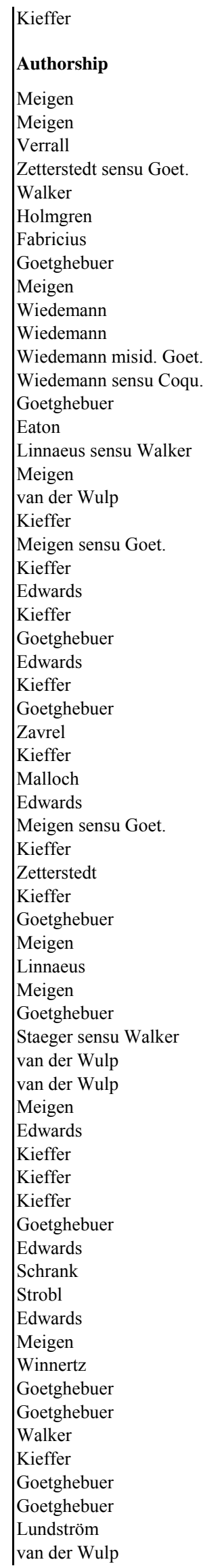

\begin{tabular}{|c|c|}
\hline Orthocladius & Spaniotoma (Psectrocladius) \\
\hline $\begin{array}{l}\text { Genus in original } \\
\text { combination }\end{array}$ & $\begin{array}{l}\text { Genus-group name(s) } \\
\text { stated or implied by Edwards }\end{array}$ \\
\hline Chironomus & Chironomus (Chironomus) \\
\hline Chironomus & Cricotopus \\
\hline Cricotopus & Cricotopus \\
\hline Chironomus & Chironomus (Polypedilum) \\
\hline Chironomus & Chironomus (Chironomus) \\
\hline Chironomus & Spaniotoma (Limnophyes) \\
\hline Chironomus & Anatopynia \\
\hline Camptocladius & Spaniotoma (Limnophyes) \\
\hline Tanypus & Tanypus \\
\hline Chironomus & Pentapedilum (Phaenopsectra) \\
\hline Chironomus & Chironomus \\
\hline Chironomus & Tanytarsus (Tanytarsus) \\
\hline Chironomus & Tanytarsus \\
\hline Camptocladius & Spaniotoma (Limnophyes) \\
\hline Limnophyes & Spaniotoma (Limnophyes) \\
\hline Tipula & Chironomus \\
\hline Tanypus & Pentaneura \\
\hline Tanypus & Pentaneura \\
\hline Polypedilum & Chironomus (Polypedilum) \\
\hline Chironomus & Chironomus \\
\hline Tanypus & Pentaneura \\
\hline Spaniotoma & Spaniotoma (Smittia) \\
\hline Tanypus & Anatopynia \\
\hline Micropsectra & Tanytarsus (Micropsectra) \\
\hline Tanytarsus & Tanytarsus (Tanytarsus) \\
\hline Nilotanypus & Nilotanypus \\
\hline Tanytarsus & Tanytarsus (Micropsectra) \\
\hline Phaenocladius & Symbiocladius \\
\hline Orthocladius & Spaniotoma (Orthocladius) \\
\hline Pseudochironomus & Pseudochironomus \\
\hline Tanytarsus & Tanytarsus (Tanytarsus) \\
\hline Chironomus & Chironomus (Chironomus) \\
\hline Orthocladius & Spaniotoma (Orthocladius) \\
\hline Chironomus & Chironomus (Stictochironomus) \\
\hline Cryptochironomus & Chironomus (Chironomus) \\
\hline Chironomus & Chironomus (Chironomus) \\
\hline Chironomus & Spaniotoma (Orthocladius) \\
\hline Tipula & Chironomus (Endochironomus) \\
\hline Chironomus & Spaniotoma (Trichocladius) \\
\hline Tanytarsus & Tanytarsus (Tanytarsus) \\
\hline Chironomus & Chironomus \\
\hline Tanypus & Tanypus \\
\hline Tanypus & Procladius (Psilotanypus) \\
\hline Tanypus & Pentaneura \\
\hline Chironomus & Chironomus (Polypedilum) \\
\hline Tanypus & Procladius (Procladius) \\
\hline Trichocladius & Spaniotoma (Trichocladius) \\
\hline Chironomus & Chironomus (Chironomus) \\
\hline Tanytarsus & Tanytarsus (Stempellina) \\
\hline Tanytarsus & Tanytarsus (Tanytarsus) \\
\hline Tipula & Chironomus (Polypedilum) \\
\hline Tanypus & Pentaneura \\
\hline Spaniotoma & Spaniotoma (Smittia) \\
\hline Chironomus & Prodiamesa \\
\hline Corynoneura & Corynoneura (Corynoneura) \\
\hline Tanytarsus & Tanytarsus (Tanytarsus) \\
\hline Tanytarsus & Pentapedilum (Phaenopsectra) \\
\hline Chironomus & Chironomus (Microtendipes) \\
\hline Dactylocladius & Spaniotoma (Orthocladius) \\
\hline Chironomus & Chironomus (Glyptotendipes) \\
\hline Tanytarsus & Tanytarsus (Micropsectra) \\
\hline Arctomyia & Arctomyia \\
\hline Chironomus & Tanytarsus (Tanytarsus) \\
\hline
\end{tabular}

331,334

Page numbers

388

319

$320 \mathrm{k}, 321$

403

381f, 386

$355 \mathrm{k}, 356$

291, 298

356

284, 299

375

406, 411

410

375

355

$280,355,355 \mathrm{k}$

418

295

294

$401 \mathrm{k}, 402$

402

292

352f, 362, 368f, $428 f$

298

408

411, $412 \mathrm{k}$

294

408

309

$345,345 \mathrm{k}$

377

412k, 413

$383 \mathrm{k}, 384$

345

401

390

385

318, 339f, 345k, 345, 347

379, 380, 393, 394

329

418

418

300

302

292

398f, 402k, 404

301

328

384

417f, 419, 420, 421

416, $417 f$

401k, 402

293, 294

363

307

280, 352f, 368f, 369

415

375,411

399

337

393

408

357

375, 406, 415 


\begin{tabular}{|c|c|c|c|c|}
\hline sima & Edwards & Spaniotoma & |Spaniotoma (Orthocladius) & $340 \mathrm{k}, 342$ \\
\hline similis & Goetghebuer & Cricotopus & Cricotopus & $320 \mathrm{k}, 321,322$ \\
\hline sinuosus & Kieffer & Trichocladius & Spaniotoma (Smittia) & 360 \\
\hline skirwithensis & Edwards & Spaniotoma & Spaniotoma (Trichocladius) & $329,329 f$ \\
\hline sordens & van der Wulp & Tanytarsus & Pentapedilum (Pentapedilum) & $310,374 f, 376$ \\
\hline sordidella & Zetterstedt & Chironomus & Spaniotoma (Psectrocladius) & $327,332 \mathrm{k}, 334,335$ \\
\hline sordidellus & Zetterstedt misid. Kieffer & Chironomus & Orthocladius & 335 \\
\hline sordidus & Zetterstedt & Tanypus & Pentaneura & 293 \\
\hline speciosus & Goetghebuer & Cricotopus & Cricotopus & 319k, 320 \\
\hline sphagnicola & Kieffer & Isoplastus & Prosisoplastus & 296 \\
\hline spitzbergensis & Kieffer & Psilodiamesa & Psilodiamesa & 305 \\
\hline stercorarius & De Geer & Tipula & Spaniotoma (Smittia) & $335,343,362$ \\
\hline stercorarius & De Geer & Tipula & Camptocladius & 357 \\
\hline stercorarius & De Geer misid. v.d.Wulp & Tipula & Orthocladius & 335 \\
\hline sticticus & Fabricius & Tipula & Chironomus (Stictochironomus) & 401 \\
\hline stigmaticus & Philippi & Podonomus & Podonomus & 295,297 \\
\hline stratiotale & Kieffer & Pentapedilum & Pentapedilum (Pentapedilum) & 376 \\
\hline stratiotis & Kieffer & Psectrotanypus & Spaniotoma (Psectrocladius) & $332 \mathrm{k}, 334$ \\
\hline stylatus & Kieffer & Metriocnemus & Metriocnemus & 315 \\
\hline subaequalis & Goetghebuer & Tanytarsus & Tanytarsus (Tanytarsus) & 415 \\
\hline subaprilinus & Kieffer & Chironomus & Chironomus (Chironomus) & 382 \\
\hline subcoeruleus & Edwards & Cricotopus & Cricotopus & 323 \\
\hline subincurvatus & Goetghebuer & Tanypus & Pentaneura & 290 \\
\hline subnitens & Goetghebuer & Micropsectra & Tanytarsus (Micropsectra) & 408 \\
\hline subnudus & Edwards & Metriocnemus & Metriocnemus & 314f, 316, 428f \\
\hline subtenuis & Kieffer & Tanypus & Anatopynia & 298 \\
\hline subvernalis & Edwards & Spaniotoma & Spaniotoma (Orthocladius) & 339f, 340k, 341 \\
\hline subviridis & Goetghebuer & Tanytarsus & Tanytarsus (Micropsectra) & 407 \\
\hline suecica & Kieffer & Dactylocladius & Spaniotoma (Orthocladius) & $337,337 \mathrm{k}, 339 \mathrm{f}$ \\
\hline suecicus & Kieffer & Trichocladius & Cricotopus & 323 \\
\hline supplicans & Meigen & Chironomus & Chironomus (Chironomus) & 388 \\
\hline sylvaticus & van der Wulp sensu Goet. & Chironomus & Tanytarsus (Tanytarsus) & 418 \\
\hline sylvestris & Fabricius & Tipula & Cricotopus & $318,319,319 \mathrm{k}$ \\
\hline sylvestris & Goetghebuer & Brillia & Brillia & 310 \\
\hline tarsalis & Walker & Chironomus & Chironomus (Microtendipes) & $397,397 \mathrm{k}$ \\
\hline tendens & Fabricius & Tipula & Chironomus (Endochironomus) & 374f, 394 \\
\hline tendipediforme & Goetghebuer & Tanytarsus & Pentapedilum (Kiefferulus) & 375 \\
\hline tenellulus & Goetghebuer & Tanytarsus & Tanytarsus (Tanytarsus) & 374f, 407, 411 \\
\hline tenellus & Zetterstedt & Chironomus & Pentapedilum (Phaenopsectra) & 375 \\
\hline tener & Kieffer & Chironomus & Chironomus (Chironomus) & 390 \\
\hline tentans & Fabricius & Chironomus & Chironomus (Chironomus) & 381f, 382 \\
\hline tenuis & Meigen & Chironomus & Tanytarsus (Tanytarsus) & 409 \\
\hline tenuis & Meigen sensu Goet. & Chironomus & Tanytarsus (Tanytarsus) & 410 \\
\hline terminalis & Kieffer & Syntanytarsus & Tanytarsus (Micropsectra) & 407 \\
\hline tetratomus & Kieffer & Tanytarsus & Tanytarsus (Micropsectra) & 407 \\
\hline thalassophila & Goetghebuer & Camptocladius & Spaniotoma (Smittia) & $360 \mathrm{k}, 362,371$ \\
\hline thienemanni & Kieffer & Orthocladius & Spaniotoma (Orthocladius) & 339f, 344k, 345 \\
\hline thummi & Kieffer & Tendipes & Chironomus (Chironomus) & 384 \\
\hline tibialis & Meigen & Chironomus & Cricotopus & $320 \mathrm{k}, 323$ \\
\hline tonnoiri & Goetghebuer & Tanytarsus & Tanytarsus (Micropsectra) & 407 \\
\hline tonsa & Haliday & Diamesa & Diamesa & 304 \\
\hline tremulus & Linnaeus & Tipula & Cricotopus & $320,320 \mathrm{k}$ \\
\hline triangulifer & Kieffer & Metriocnemus & Metriocnemus & 313,313 \\
\hline triannulatus & Goetghebuer & Tanypus & Pentaneura & 292 \\
\hline triannulatus & Macquart & Chironomus & Cricotopus & $320 \mathrm{k}, 323$ \\
\hline triannulatus & Macquart misid. Goet. & Chironomus & Cricotopus & 321,323 \\
\hline trichopus & Walker & Chironomus & Chironomus (Endochironomus) & 394 \\
\hline tricinctus & Meigen & Chironomus & Cricotopus & $319,319 \mathrm{k}$ \\
\hline tricolor & Kieffer & Cryptochironomus & Chironomus (Chironomus) & 390 \\
\hline trifascia & Edwards & Cricotopus & Cricotopus & $320 \mathrm{k}, 322,322 \mathrm{f}$ \\
\hline trifasciatus & Panzer & Chironomus & Cricotopus & 319, 319k \\
\hline trifascipennis & Zetterstedt & Tanypus & Psectrotanypus & 297 \\
\hline trifascipennis & Zetterstedt & Tanypus & Anatopynia & 299 \\
\hline trifida & Kieffer & Orthocladius & Spaniotoma & 340 \\
\hline trilobata & Edwards & Spaniotoma & Spaniotoma (Smittia) & $352 \mathrm{f}, 363 \mathrm{k}, \mathbf{3 6 4}, 368 \mathrm{f}, 428 \mathrm{f}$ \\
\hline trinotatus & Kieffer & Dactylocladius & Spaniotoma (Orthocladius) & 337 \\
\hline tripilata & Edwards & Spaniotoma & Spaniotoma (Orthocladius) & $339 f, 347 \mathrm{k}, \mathbf{3 4 8}, 368 \mathrm{f}, 428 \mathrm{f}$ \\
\hline
\end{tabular}




\begin{tabular}{|c|c|}
\hline tristellus & Edwards \\
\hline Species-group name & Authorship \\
\hline tristis & Kieffer \\
\hline tristis & Wiedemann \\
\hline tritomus & Kieffer \\
\hline tritum & Walker \\
\hline truncatus & Kieffer \\
\hline truncorum & Goetghebuer \\
\hline tuberculata & Edwards \\
\hline turfacea & Kieffer \\
\hline typhon & Haliday \\
\hline uncinatum & Goetghebuer \\
\hline unifasciatus & Macquart \\
\hline unifascipennis & Zetterstedt \\
\hline vagans & Meigen \\
\hline vanderwulpi & Edwards \\
\hline variabilis & Staeger \\
\hline varians & Staeger \\
\hline varius & Fabricius \\
\hline varius & Fabricius \\
\hline varus & Goetghebuer \\
\hline venustus & Staeger \\
\hline vernalis & Goetghebuer \\
\hline verralli & Edwards \\
\hline verralli & Goetghebuer \\
\hline verruculosus & Goetghebuer \\
\hline viator & Kieffer \\
\hline vilipennis & Kieffer \\
\hline virescens & Meigen \\
\hline virescens & Meigen sensu Goet. \\
\hline viridanus & Ruthe sensu Goet. \\
\hline viridicollis & van der Wulp \\
\hline viridis & Macquart sensu Goet. \\
\hline viridulus & Linnaeus sensu Goet. \\
\hline virtunensis & Goetghebuer \\
\hline vitellinus & Kieffer \\
\hline vitiosus & Goetghebuer \\
\hline vitripennis & Meigen \\
\hline vitripennis & Meigen \\
\hline vittata & Edwards \\
\hline vulneratus & Zetterstedt \\
\hline waltli (incorr. subs. spellg.) & Meigen \\
\hline woodi & Edwards \\
\hline xanthogyne & Edwards \\
\hline xenolabis & Kieffer \\
\hline zavreli & Kieffer \\
\hline zonarius & Walker \\
\hline
\end{tabular}

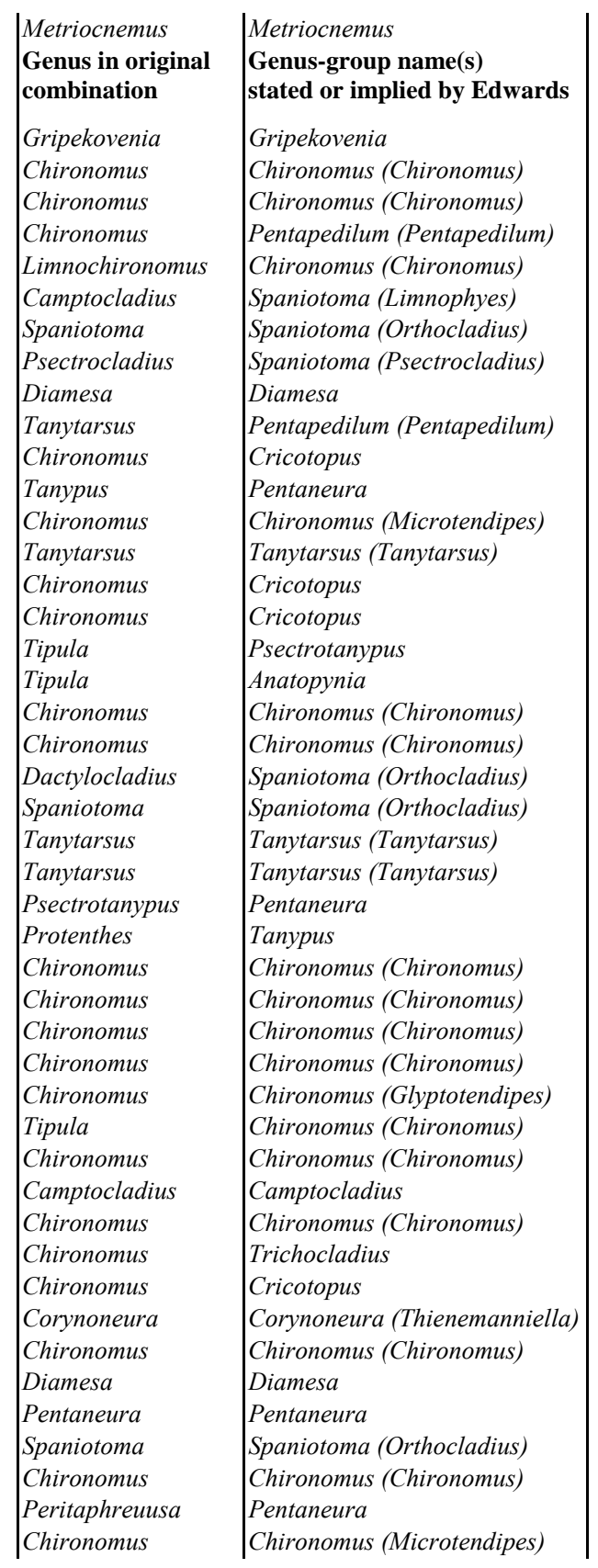

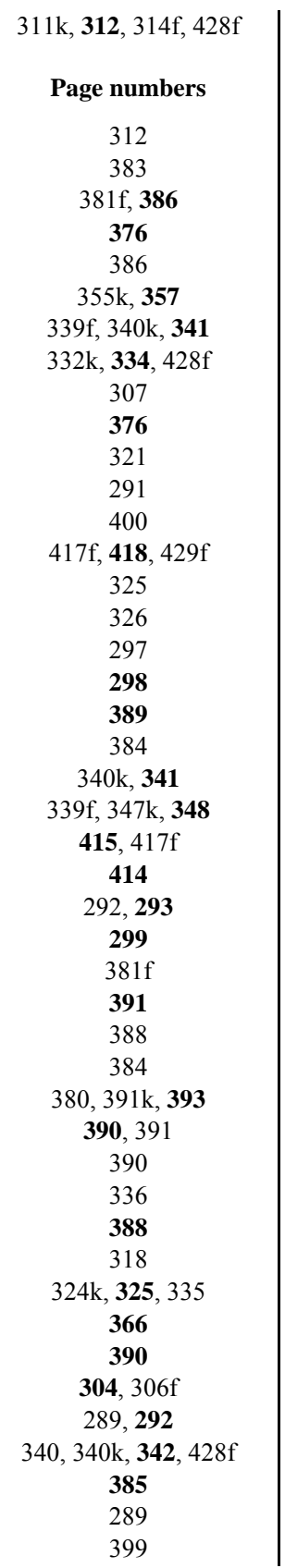

\section{References}

EDWARDS, F. W. (1929): British non-biting midges (Diptera, Chironomidae). - Trans. Ent. Soc. London 77: 279-430.

GOETGHEBUER, M. (1940-1950): Tendipedidae (Chironomidae). f) Subfamilie Orthocladiinae. A. Die Imagines. Pp. 1-208, 24 pls. in: LINDNER, E. (ed.), Die Fliegen der palaearktischen Region, vol. 3, part 13g. Stuttgart, Schweizerbart.

GOETGHEBUER, M. \& LeNZ, F. (1937-1962): Tendipedidae (Chironomidae). b) Subfamilie

Tendipedinae (Chironominae). Pp. 1-260, 23 pls. in: Lindner, E. (ed.), Die Fliegen der palaearktischen Region, vol. 3, part 13c. Stuttgart, Schweizerbart.

SPIES, M. (2001): When is a nomen really dubium? Toward real stability in chironomid taxonomy through better symbiosis with the classic collections. - Chironomus 14: 7-10.

SPIES, M. \& Słther, O. A. (2004): Notes and recommendations on taxonomy and nomenclature of Chironomidae (Diptera). Zootaxa 752: 1-90. 